Introduction: Parathyroid adenoma with prominent lymphocytic infiltration is rare, with only 12 cases reported in the English scientific literature. All these cases were not related to synchronous autoimmune or IgG4-related disease and presented clinically with primary hyperparathyroidism.

Case presentation: A 65-year-old woman presented with hypercalcemia and a neck mass, suggestive of parathyroid adenoma. She underwent parathyroidectomy and histopathological examination of the specimen showed a hypercellular parathyroid nodule comprising mature lymphoid cells admixed with parathyroidal chief cells. A comprehensive immunohistochemistry panel confirmed the benign nature of the lesion. The patient remains asymptomatic a year after the surgery.

Discussion: Parathyroid adenoma with prominent lymphocytic may be misdiagnosed as a lymph node at frozen section or cytology smears by an unsuspecting pathologist or cytologist, if the lymphoid component is predominant on the slide examined. Additionally, the morphology could be misinterpreted as a metastasis of parathyroid carcinoma into a peri-thyroidal lymph node. For this distinction, correlation with clinical and radiological findings is crucial and clues to adenoma include presence of a rim of uninvolved parathyroid parenchyma, low mitotic activity $(<5 \%)$ and positive staining for parafibromin, bcl-2 and MDM2 on immunohistochemistry.

\section{EOSINOPHILIC SOLID AND CYSTIC RENAL CELL CARCINOMA WITH MONOSOMY OF CHROMOSOMES $X$ AND 3}

Nassim Saremi $^{1,2}$, Admire Matsika ${ }^{1,3}$, Bhuvana Srinivasan ${ }^{1,3}$ ${ }^{1}$ Anatomical Pathology Department, Mater Health, South Brisbane, Qld, Australia; ${ }^{2}$ Pathology Queensland, Central Laboratory, Queensland Health, Herston, Qld, Australia; and ${ }^{3}$ School of Biomedical Sciences, University of Queensland, Qld, Australia

Introduction: Eosinophilic solid and cystic renal cell carcinoma (ESC-RCC) is a recently described sporadic counterpart to tuberous sclerosis complex (TSC)-associated RCC that is not yet included in the 2016 World Health Organization (WHO) classification of renal neoplasms. The first series was reported in 2016 and, to date, just over 60 cases have been reported in the literature. The tumour is often asymptomatic and almost exclusive to female patients with a median age of 57 years. ESC-RCC constitutes approximately $0.2 \%$ of all renal cell carcinomas and most cases reported have shown a better prognosis than clear cell renal cell carcinoma. About $10 \%$ of reported cases have metastasised with four of them dying of the malignancy.

Case report: A 63-year-old woman presented with an incidental $40 \mathrm{~mm}$, ill-defined nodule in the lower pole of the right kidney. Partial nephrectomy was performed and gross examination of the tumour showed a well-circumscribed neoplasm with solid-cystic cut surface. Microscopy revealed acinar and nested architecture with the neoplastic cells showing intracytoplasmic stippling ('Leishmania bodies-like') within the deeply eosinophilic cytoplasm. Macro- and micro-cysts in other fields were lined by hobnailed, eosinophilic tumour cells. Immunohistochemically, the neoplasm showed strong and diffuse staining for CK20 and AE1/AE3. CK7 was negative. Karyotyping of tumour cells showed previously unreported monosomy of chromosomes $\mathrm{X}$ and 3. The patient remains alive and well, without evidence of disease progression a year after surgery.

\section{CLINICAL UTILITY OF THE TFE3 BREAK-APART FLUORESCENCE IN SITU HYBRIDISATION (FISH) ASSAY IN MALIGNANT TUMOURS}

\author{
T. H. Lim ${ }^{1}$, S. T. A. Lim ${ }^{1}$, Y. S. Y. Yeap ${ }^{1}$, Y. J. $\mathrm{Ng}^{1}$,

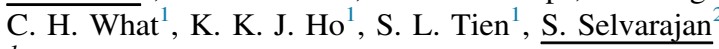 \\ ${ }^{1}$ Department of Molecular Pathology, Singapore General \\ Hospital, Singapore; and ${ }^{2}$ Department of Anatomical \\ Pathology, Singapore General Hospital, Singapore
}

TFE3 break-apart FISH probe, located on Xp11.23 is designed to detect TFE3 gene rearrangements in many tumours including renal cell carcinomas (RCC) and alveolar soft part sarcoma (ASPS), and some variants of perivascular epitheloid cell neoplasm (PEComa). FISH test may be deployed as an adjunct tool for diagnosis. Generally, the FISH probe will give a typical FISH signal pattern but unusual patterns can also be encountered. We aimed to evaluate the break-apart FISH patterns and incidence of TFE3 gene rearrangements in soft tissue tumours such as RCC and ASPS.

As the morphological assessment may have overlapping features, TFE3 FISH test is a useful ancillary tool in the confirmation or exclusion of differential diagnosis.

\section{MALIGNANT SOLITARY FIBROUS TUMOUR OF UTERINE CERVIX, MIMICKING HIGH GRADE ENDOMETRIAL STROMAL SARCOMA}

Pooja Singhal, Raghwa Sharma, Spinderjeet Samra Tissue Pathology and Diagnostic Oncology, ICPMR, Westmead Hospital, NSW, Australia

Solitary fibrous tumour (SFT) is an uncommon mesenchymal neoplasm of fibroblastic origin which can be found in any organ, however, its occurrence in the female genital tract (FGT) is extremely rare. To date, around 50 cases of SFTs originating in FGT have been reported (as case report or small case series) of which 12 are in the uterus and 5 in the cervix. These are rare tumours and should be considered in the differential diagnosis of spindle cell lesion in the female genital tract when the immunohistochemistry is not supportive of common lesions. We are reporting a case of a malignant SFT involving primarily the cervix and extending into the isthmic uterus, involving the bladder, presenting with recurrence and distant metastasis to the lung and mesentery.

\section{OLFACTORY NEUROBLASTOMA WITH DIVERGENT DIFFERENTIATION}

Pooja Singhal, Narinder Singh, Spinderjeet Samra, Hedley Coleman

Tissue Pathology and Diagnostic Oncology, ICPMR, Westmead Hospital, NSW, Australia

Olfactory neuroblastoma (ONB) is an uncommon malignant neoplasm of the nasal cavity that arises from olfactory epithelium. Rare cases of focal histologic changes of divergent cell populations have been reported in ONB. However, these changes are seen mainly in tumours treated with chemo radiation or with distant metastases. 
To date, only four cases of ONB associated with non-neuroendocrine tumours have been reported. We describe a case of ONB with divergent differentiation in a 68-year-old male who presented with a nasal cavity mass extending into the brain and base of the skull. The pathology showed the presence of two distinct cell populations showing features of high grade ONB and poorly differentiated / undifferentiated carcinoma with focal abrupt keratinisation. The differential diagnosis included NUT carcinoma and teratocarcinoma which were excluded since the immune markers and morphology were not supportive.

\section{EPITHELIOID SCHWANNOMA - A RARE VARIANT OF A COMMON LESION}

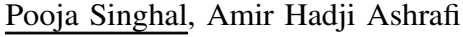 \\ Anatomical Pathology, Nepean Hospital, NSW, Australia
}

Epithelioid schwannoma is a rare variant of schwannoma arising in dermis or subcutis of limbs as a circumscribed lesion. Less than 100 cases of epithelioid schwannoma have been reported so far. They are considered benign despite the presence of atypical features such as nuclear atypia.

We present a case of a 26-year-old female who presented with a left posterior thigh lump with the clinical diagnosis of lipoma. The microscopic examination, however, is in keeping with epithelioid schwannoma.

This case demonstrates a very rare variant of a common lesion which can mimic few other soft tissue tumours including some malignant tumours. Hence, this may help pathologists to become familiar with this entity and avoid potential wrong diagnoses.

\section{NODULAR LYMPHOCYTE PREDOMINANT HODGKIN LYMPHOMA - CASE REPORT IN A 6 YEAR OLD}

Mathuranthakan Sinnathamby, Deepali Kamra

Histopathology Department, Dorevitch Pathology, Albury, Australia

Nodular lymphocyte predominant Hodgkin lymphoma (NLPHL) is a rare subtype of Hodgkin lymphoma in children accounting for $10-20 \%$ of paediatric cases of Hodgkin lymphoma. In the paediatric population the reported median age at diagnosis is 13 years with a male preponderance and patients typically presenting with low stage disease. ${ }^{1-3}$ It has a generally good prognosis with less aggressive treatment strategies compared to classical Hodgkin lymphoma. Histologically it is characterised by the presence of atypical Reed-Sternberg cells known as the lymphocytic and histiocytic variant $(\mathrm{L} \& \mathrm{H})$ which have a vesicular polylobulated nucleus which is referred to as popcorn cells. A 6-year-old boy presented with a single rubbery lymph node mass in the neck with no other systemic symptoms. An excisional biopsy of the node was performed. Microscopically there was a nodular proliferation of small lymphocytes and scattered popcorn cells with the typical morphology and immunohistochemistry of L\&H cells. No further treatment was performed and the patient was for regular follow up. One year later the patient is well and free from recurrence.

\section{References}

1. Eichenauer DA, Plütschow A, Fuchs M, et al. Long-term course of patients with stage IA nodular lymphocyte-predominant Hodgkin lymphoma: a report from the German Hodgkin Study Group. J Clin Oncol 2015; 33: 2857-62.
2. Nogová L, Reineke T, Brillant C, et al. Lymphocyte-predominant and classical Hodgkin's lymphoma: a comprehensive analysis from the German Hodgkin Study Group. J Clin Oncol 2008; 26: 434-9.

3. Shankar A, Daw S. Nodular lymphocyte predominant Hodgkin lymphoma in children and adolescents - a comprehensive review of biology, clinical course and treatment options. Br J Haematol 2012; 159: 288-98

\section{ASPERGILLUS AORTITIS IN A BIOPROSTHETIC AORTIC ROOT AT POST MORTEM}

Mathuranthakan Sinnathamby, Kim Cheah

Histopathology Department, Dorevitch Pathology, Albury, Australia

Aspergillus terreus is a rare cause of endocarditis. Fungal endocarditis represents less than $2 \%$ of all cases of endocarditis and of these $20-25 \%$ are represented by Aspergillus species. ${ }^{1}$ Aspergillus terreus is especially rare with only one reported case in the literature between 1950-2010. ${ }^{2}$ Immunocompromise and previous cardiac surgery are well recognised risk factors for fungal endocarditis. ${ }^{3}$ Complications are common and include embolisation to pulmonary, ophthalmic, renal, and other arterial systems. This is a report of an 80-year-old male with a history of Aspergillus terreus aortitis treated with aortic root replacement and coronary artery bypass grafting that subsequently died of culture negative sepsis. He was found at autopsy to have extensive Aspergillus terreus endocarditis involving a bio prosthetic aortic root replacement complicated by thrombosis of the left renal artery.

\section{References}

1. Tattevin P, Revest M, Lefort A, et al. Fungal endocarditis: current challenges. Int J Antimicrob Agents 2014; 44: 290-4.

2. Kalokhe AS, Rouphael N, El Chami MF, et al. Aspergillus endocarditis: a review of the literature. Int J Infect Dis vol. 2010; 14: e1040e1047.

3. Beh YY, Lim GLH, Stewart A. Recurrent Aspergillus endocarditis in an immunocompetent patient: challenges in diagnosis and management. Res Medica 2017; 24: 1567.

\section{INVASIVE BREAST CARCINOMA OF NO SPECIAL TYPE WITH OSTEOCLAST-LIKE STROMAL GIANT CELLS: A CASE REPORT}

Carla Smith, Bhuvana Srinivasan

Department of Anatomical Pathology, Mater Hospital, Brisbane, Qld, Australia

Invasive breast carcinoma of no special type (NST) with osteoclast-like stromal giant cells (OSGCs) is a rare tumour type. The literature available for this entity is predominantly limited to case reports and small retrospective studies. The patient is a 46-year-old female who presented to her GP with a 4-month history of a palpable mass in the left breast. An abnormality seen on a mammogram was confirmed to be an invasive cancer on core biopsy. She subsequently underwent a wide local excision of the lesion and sentinel lymph node biopsy. Histopathology showed a grade 2 invasive breast carcinoma NST with OSGCs and focal lymphovascular space invasion. Sentinel lymph node biopsy demonstrated involvement of one in seven lymph nodes with minimal extra-nodal spread. Immunohistochemistry showed a strongly ER/PR positive, HER-2 negative, 